\title{
Subcritical Contraction for Improved Open-Channel Flow Measurement Accuracy with an Upward-Looking ADVM
}

\author{
Daniel J. Howes, M.ASCE ; Charles M. Burt, M.ASCE ; and Brett F. Sanders, M.ASCE
}

\begin{abstract}
Acoustic Doppler velocity meters (ADVMs) provide an alternative to more traditional flow measurement devices and procedures such as flumes, weirs, and stage rating for irrigation and drainage canals. However, the requirements for correct calibration are extensive and complex. A three-dimensional computational fluid dynamics (CFD) model was used to design a subcritical rapidly varied flow contraction that provides a consistent linear relationship between the upward-looking ADVM sample velocity and the cross-sectional average velocity in order to improve ADVM accuracy without the need for in situ calibration. CFD simulations validated the subcritical contraction in a rectangular and trapezoidal cross section by showing errors within +1.8 and $-2.2 \%$. Physical testing of the subcritical contraction coupled with an upward-looking ADVM in a large rectangular flume provided laboratory validation with measurement errors within $\pm 4 \%$ without calibration.
\end{abstract}

CE Database subject headings: Open channel flow; Subcritical flow; Flow measurement; Acoustic techniques; Doppler systems; Laboratory tests; Hydraulics.

Author keywords: Open channel flow; Subcritical flow; Flow measurement; Acoustic techniques; Doppler systems; Laboratory tests; Hydraulics.

\section{Introduction}

A pulsed acoustic Doppler velocity meter (ADVM) uses one or more acoustic transducers, which each sends and receives sound signals (Morlock et al. 2002; Styles et al. 2006). The transducer transmits an acoustic beam as a pulse of a known frequency along a narrow path. When the pulse hits sediment or air bubbles suspended in water, it scatters and some of the sound signal returns back to the transducer. The time it takes for this "return signal" or backscatter to return to the transducer depends on the distance along the beam path at which the sediment or air bubble is located. Factors affecting the resolution of the velocity measurements include ADVM operating frequency, pulse length, fixed pulse repetition frequency, and properties of the water that affect the speed of sound such as temperature and salinity (Hardcastle and Thorne 1997). The frequency of each backscatter signal has a Doppler shift (the change or shift in the original signal wave frequency due to the velocity of particles reflecting the signal back to the transducer) that is proportional to the fluid velocity (Morlock et al. 2002). The set of return signals therefore provides a set of distances and velocities at that moment measured within the limited sample area of the acoustic beam.

The most common types of signal processing used to measure water velocities are continuous (incoherent) or pulsed (coherent or profiling) (Vermeyen 2000). Continuous wave devices emit a continuous acoustic signal from a transmitter and the backscatter is measured continuously throughout the measurement range by a receiver providing the overall average velocity along the beam with no relationship to the depth. In contrast, a pulsing device sends encoded pulses along multiple beam paths and return signals are discretely measured, allowing for multiple velocity measurements along the beam at known, discrete depths (Vermeyen 2000). Pulsed ADVM devices historically have been more expensive than continuous units (Vermeyen 2000). However, pulsed wave systems provide significantly higher velocity resolution (Hardcastle 1995). Studies by Vermeyen (2000) and the Irrigation Training and Research Center (ITRC) (2005) indicate improved accuracy with noncalibrated devices using pulsed wave signals compared to devices using continuous wave.

The carrier frequency and transducer angle (beam angle) can also vary depending on the acoustic Doppler application. A typical range of acoustic Doppler operating frequencies used in a variety of water velocity measurement applications is 0.25 $10 \mathrm{MHz}$. For constant monitoring of discharge measurements in irrigation and drainage applications acoustic frequencies from 1.2 to $5 \mathrm{MHz}$ are most common (ITRC 2005; Morlock et al. 2002; Vermeyen 2000, 2005). Lower frequencies allow for a longer range of measurements typically needed in deep water or wide channel measurement locations but result in lower resolution (Simpson 2001). For shallower depths or shorter range, higher frequency signals can be used to improve measurement resolution.

The angle of the acoustic beam relative to the velocity streamlines is termed "beam angle" and is typically measured perpen- 
dicular to the streamlines. For constant monitoring applications, typical ADVM beam angles are $25^{\circ}-45^{\circ}$. The $45^{\circ}$ angle provides improved velocity measurement performance compared to the $25^{\circ}$ but the measurement range is reduced (Huhta and Ward 2003). For example, a 3-MHz acoustic transducer may have an overall range of $7 \mathrm{~m}$. If it is placed on the bottom of a channel with a beam angle of $25^{\circ}$ it can measure velocities up to a vertical depth of $6.3 \mathrm{~m}$ compared to the same transducer with a beam angle of $45^{\circ}$ measuring to a vertical depth of approximately $5 \mathrm{~m}$.

The distance from the transducer to the first measurement is called the "blanking distance." A blanking distance is required so that the pulsed wave transducer has time to switch from transmitter (sending the pulse) to receiver where it begins receiving the backscatter signal (Simpson 2001). It also serves the second function of projecting into the flow profile and starting velocity measurements outside of the obstructed velocity region caused by the ADVM itself.

ADVM installations in channels may utilize side-looking (side-mounted) configurations that sample horizontally through the cross section or upward-looking (bottom-mounted) configurations that sample upward through the cross section. Due to its superior accuracy in channels with variable flow depths, this study focuses on a pulsed upward-looking ADVM that is mounted at the centerline of the channel and uses two velocity measurement beams. One beam is angled $45^{\circ}$ (from vertical) in the upstream direction and the other is angled $45^{\circ}$ in the downstream direction. A third acoustic transducer is oriented in the vertical direction to measure the flow depth directly above the device. For this study it is assumed that the pulsed ADVM is capable of measuring every $3.4 \mathrm{~cm}$ vertically through the vertical centerline of the water surface profile. The number of individual measurements depends on the water depth.

Device software requires that information on channel geometry be input manually for the ADVM sensor to estimate discharge. Assumptions regarding velocity contours for various channel section geometries are typically provided by the manufacturer's software to convert the sample velocities into an average channel velocity. However, velocities are only measured by the ADVM within a small area of the flow cross section. Therefore, in a typical cross section, an ADVM does not provide an average cross-sectional velocity but rather only a sample of that velocity.

A calibration procedure [sometimes referred to as velocity indexing but herein termed the flow rate indexing procedure (QIP)] has been developed to address this problem of converting the sample velocity into a true average channel velocity. This procedure has been incorporated into the software run by many ADVM devices (Morlock et al. 2002; Patino and Ockerman 1997; Styles et al. 2006). At least 10 individual flow and depth conditions are recommended for QIP. It is time consuming and logistically challenging to obtain calibration data over a wide range of flow conditions.

Irregular roughness features such as vegetation and variable channel cross sections create nonuniform velocity distributions that confound efforts to implement QIP. Consequently, ADVM discharge measurement is recommended in a lined channel section positioned downstream of long (distance at least 10 times the channel width) and straight channel sections with a constant roughness to ensure UF (Styles et al. 2006). This also poses a logistical challenge and the long length of lined channel increases the cost of ADVM installations.

The objective of this study is to identify and report a channel contraction design in support of ADVM discharge measurement with a commonly used two-beam device deployed in an upwardlooking configuration. This was achieved by analyzing candidate contraction designs using computational fluid dynamics (CFD) software. Physical modeling results of the section in a laboratory flume are presented to validate the design and assess the overall discharge measurement accuracy made possible by the method.

The proposed channel contraction is designed to create a subcritical rapidly varied flow (RVF) section which stands in contrast to the lined uniform flow (UF) sections conventionally recommended for ADVM installations. Subcritical RVF locally increases the Froude number and creates a predictable velocity distribution that readily enables direct measurement of the average cross-sectional velocity with a linear indexing relationship. The primary rationale for the RVF approach over the UF approach is cost for both construction and calibration. The RVF section can be shorter in length than a UF section to attain the same level of accuracy, with potentially no need for calibration.

\section{Cal Poly ITRC Open Flume}

An open flume at the ITRC, California Polytechnic State University, San Luis Obispo (Cal Poly) was used to support design of the proposed RVF section. First, CFD software was calibrated to flow measurements taken in the Cal Poly flume without the RVF section in place. Subsequently, the CFD-based contraction design was tested by making discharge measurements in the flume with the proposed contraction design in place.

The Cal Poly ITRC flume has dimensions of $1.215 \mathrm{~m}$ in width by $1.215 \mathrm{~m}$ in depth by $86 \mathrm{~m}$ in length. The bottom slope of the painted steel flume is 0.002 . Flume components are capable of handling flow rates up to $0.85 \mathrm{~m}^{3} / \mathrm{s}$. The testing region of the flume is approximately $54 \mathrm{~m}$ long. The start point of the testing region was just downstream of a flow conditioner consisting of a 1-m-long honeycomb of 3-in. diameter PVC pipes. At the downstream end of the flume a vertical weir is used to fix the water level in the testing region.

The Cal Poly flume uses a recirculation facility to achieve high flow rates in the flume. The discharge is measured in real time by a calibrated 0.76- $\mathrm{m}$ diameter McCrometer magmeter installed in a long straight section of pipe feeding the head of the flume. The discharge into the flume is regulated through a valve at the flume entrance.

\section{CFD Design of Channel Contraction}

\section{Theoretical Background}

Contractions (bottom or side or both) are commonly used to promote critical flow, which is useful for flow measurement purposes because the flow rate is uniquely determined by the depth (Chow 1959; Clemmens and Bos 1992). Critical flow corresponds to a Froude number $(F)$ of unity (1.0) which in a rectangular channel is given by Eq. (1)

$$
\mathrm{F}_{\text {critical }}=\frac{V}{\sqrt{g h}}=1.0
$$

where $V=$ cross-sectional average velocity; $g=$ acceleration of gravity; and $h=$ flow depth.

Critical flow devices for open channels include broad crested weirs, computable or Replogle's flumes, and Parshall's flumes 
(Clemmens et al. 1984; Replogle 2002). These devices can be robust and accurate if properly designed, constructed, and maintained. Unfortunately, the necessary head is not always available, transitions to supercritical flow can create erosion problems, many designs trap sediment, and flumes can be difficult to configure for a wide range of flow rates and water levels.

Our analysis centers on a channel contraction that promotes RVF but not critical flow. We seek to transition subcritical channel flow conditions to a larger Froude number without exceeding unity (1.0). The contraction promotes convective acceleration of the fluid that distorts the velocity profile in a predictable manner and consequently facilitates measurement of the average crosssectional velocity using the ADVM. That is, we obtain a consistent relationship between the ADVM measured velocity and the cross-sectional average velocity to minimize the necessary calibration. Velocity profile distortion is beneficial as long as the distortion is symmetric about the vertical centerline axis. This means that the contraction must be placed in a channel section that has undistorted velocity profiles along the horizontal axis.

A channel contraction was designed with the support of a commercial CFD program flow three dimensions (Flow Science, Inc., Santa Fe, N.M.). CFD was used to evaluate several configurations of the contraction focusing on the inlet shape, the throat length, the throat-to-channel width ratio, and the outlet shape. In each case, flow through the contraction was simulated using CFD, and ADVM measurements were simulated by sampling the predicted velocity distribution consistent with the location and orientation of the ADVM beams.

\section{CFD Model Calibration}

In order to utilize CFD to design a channel contraction, a preliminary study was needed to identify the mesh resolution and model parameters used to predict velocities with an accuracy that would be sufficient from an ADVM flow measurement perspective. The study involved calibration of the model and accuracy validation by comparing CFD results with physical measurements in the $\mathrm{Cal}$ Poly ITRC flume. The flume was configured without a contraction in place. Using three-dimensional solid objects in the computer aided design (CAD) software AutoCAD (Autodesk, San Rafael, Calif.), the inner flume area was created with the same overall dimensions as the Cal Poly flume including width, length, height, and slope. The basic flume was then imported into the CFD model.

A nested grid was used to depict the flume. The outer regular grid size for the simulated flume was $0.03 \mathrm{~m}$ in the $x$-direction (width of the flume), $0.09 \mathrm{~m}$ in the $y$-direction (flow direction), and $0.06 \mathrm{~m}$ in the $z$-direction (depth). The contraction section required additional resolution to appropriately render the components and account for the turbulence in the region. A nested block (more detailed grid set inside of the original grid) was used with a grid size of $50 \%$ of the original size. The mesh size for the nested block containing the subcritical RVF contraction, and approximately $1-\mathrm{m}$ upstream and downstream of the contraction, was $0.015 \mathrm{~m}$ in the $x$-direction, $0.045 \mathrm{~m}$ in the $y$-direction, and $0.03 \mathrm{~m}$ in the $z$-direction. This grid size was found to provide sufficient accuracy and computational speed using approximately 765,000 active cells after experimenting with alternative grid sizes.

At the upstream boundary, the velocity was specified uniformly parallel to the channel bottom and centerline to achieve the designed flow rate. As with the actual Cal Poly ITRC flume a sharp crested weir was incorporated in the flume geometry used for CFD modeling to fix the water level in the channel. The weir was placed $8 \mathrm{~m}$ from the downstream end of the flume and sufficiently far from the test section $(30 \mathrm{~m})$ that it did not impact the targeted velocity distribution.

No-slip wall boundary conditions were set for the walls and channel bottom. The renormalization-group turbulence model was used with a turbulent mixing length equal to $6-7 \%$ of the hydraulic diameter of the flow through the channel. The turbulent mixing length is used to limit the dissipation so that turbulent viscosity does not become excessively large. The viscous stress was solved explicitly.

Calibration of the surface roughness in the CFD model involved comparing a uniform grid of 36 cross-sectional velocity measurements from the Cal Poly ITRC flume at multiple depth, flow rate, and turbulence scenarios to the model results under the same conditions. Velocity values were extracted from the CFD simulation results at the same location as the measurements taken in the Cal Poly ITRC flume. Iteratively the surface roughness was adjusted until the CFD and flume velocities matched. The calibration effort resulted in a surface roughness $\left(k_{s}\right)$ of $0.0002 \mathrm{~m}$ for the flume walls and bottom, which is consistent with the painted steel Cal Poly ITRC flume.

In addition to the calibration measurements, cross-sectional velocity samples were measured in the Cal Poly ITRC flume under different flow rate, water depth, and turbulence scenarios. These measurements were used to validate the CFD model. An evenly spaced grid of 36 velocity samples was measured using a SonTek/YSI FlowTracker Handheld acoustic Doppler velocimeter. These velocity measurements were compared to the velocities extracted from the same location in the CFD model flume using the same flow and depth scenarios. Results of the initial validation procedure showed the coefficient of variation of the root mean squared error (CVRMSE) (CVRMSE is the RMSE of the 36 velocities divided by the actual average cross-sectional velocity at the measurement location) for scenarios similar to those studied in this paper to be between 4 and $8 \%$. A portion of the error between the simulated and the physical flume velocities is related to the inability of any CFD to completely resolve turbulence at scales that are computationally feasible. Another portion of the uncertainty is related to measurement errors such as the errors related to the measurement device, fluctuations in velocities at each sample point, and errors related to measurement procedure. Overall the accuracy of each point velocity measurement in the Cal Poly ITRC flume is believed to be within $\pm 3 \%$. Unlike the CFD simulated flume, the sides and bottom of the Cal Poly ITRC flume are not perfectly flat so some variations in velocity between the CFD flume and the physical flume are expected. It was concluded that the CFD simulated flume provided sufficient resolution and was representative of the physical Cal Poly ITRC flume allowing CFD modeling to be used for subcritical RVF contraction design.

Several aspects of the contraction design are examined in the following section. In each case, the geometry of the contraction within the flume is modified but model parameters are otherwise the same as above.

\section{Inlet Design}

It was initially hypothesized that poor inlet conditions, causing flow separation at the entrance to the contraction throat, would not provide ideal velocity distributions for flow measurement. The flow separation caused by the poor inlet condition could lead to inconsistency in velocity measurements over a range of dis- 


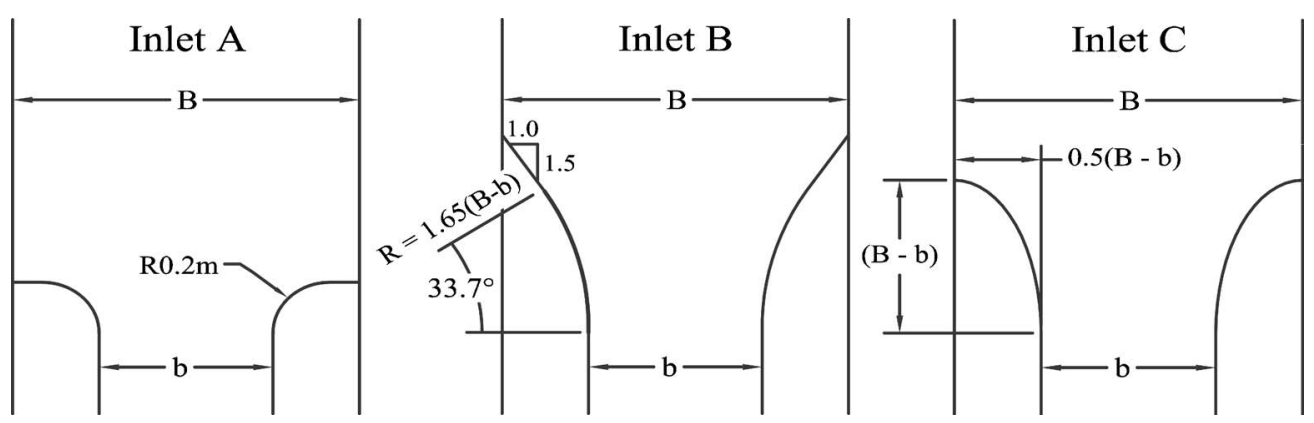

Fig. 1. Three inlets analyzed for the subcritical contraction

charges and depths caused by excessive turbulence and variable flow separation. This was tested using three contraction inlet designs shown in Fig. 1. Inlet $\mathrm{A}$ is a simple inlet condition with a $0.2-\mathrm{m}$ radius rounded entrance. Inlet $\mathrm{B}$ is a combination of straight and rounded entrances described by Smith (1967). Inlet C is an elliptical entrance shown by Montes (1998). Inlets B and C were selected based on low energy loss coefficients and their relatively short length compared to alternative designs (Montes 1998).

The objective was to maintain subcritical flow through the contraction; therefore, scenarios where critical or supercritical flow $(F \geq 1)$ would be attained were not simulated. The contraction ratio $(\mathrm{CR})$ of 0.5 [the ratio of the width of the contraction opening $(b)$ to the channel width upstream of the contraction $(B)]$ was analyzed for this portion of the study. Each inlet was simulated with a contraction throat length of $4 \mathrm{~m}$ and a simple rounded exit transition with a radius equal to $0.5 b$. The CFD simulation scenarios for the inlet design testing included discharges of 0.283 , $0.425,0.566$, and $0.708 \mathrm{~m}^{3} / \mathrm{s}$ at nominal depths of 1 and $0.65 \mathrm{~m}$ as well as $0.283 \mathrm{~m}^{3} / \mathrm{s}$ at a nominal depth of $0.35 \mathrm{~m}$.

For these initial design components, examination of the general velocity distribution was used to examine the effects of contraction components on the overall velocity profile. Later, testing involved the use of a hypothetical ADVM beams to relate the sampled velocity to the cross-sectional average velocity based on the design configuration. Velocity and water level data from the CFD simulations were examined at the cross section M2, the entrance to the contraction throat, shown in Fig. 2. An evenly spaced 36-point grid of velocity data was extracted from the CFD

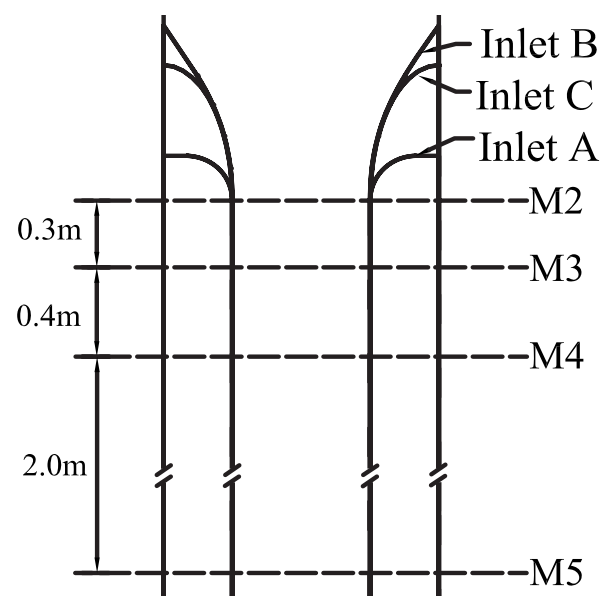

Fig. 2. Top view of the contraction showing measurement locations examined for inlet and throat length design model for each scenario. The average cross-sectional velocity $(V)$ was calculated from the discharge entering the CFD flume divided by the wetted cross-sectional area $(A)$ at M2. The Froude number (F) was calculated at M2 based on $V$ and the flow depth (h) at M2 using Eq. (1).

For application purposes, the contraction inlet should be simple to construct and will limit flow separation from the contraction walls at the entrance. Flow separation near the entrance acts as a flow restriction and can be identified by higher velocities in this region. A comparison of the ratio of maximum sampled velocity $\left(U_{\max }\right)$ to $V$ at $\mathrm{M} 2$ against the Froude number computed at M2 $\left(F_{2}\right)$ is shown in Fig. 3 for the three inlet designs.

The maximum velocity just downstream of Inlet A was higher than both Inlets B and C, indicating flow separation from the wall. The relationship $U_{\max } / V$ for Inlets $\mathrm{B}$ and $\mathrm{C}$ were similar, although the results for Inlet B seemed to indicate slight improvement over Inlet $\mathrm{C}$ for most scenarios. The ratio of $U_{\max } / V$ was more consistent for both Inlets $\mathrm{B}$ and $\mathrm{C}$ over the range of $\mathrm{F}$ tested compared to Inlet A.

Inlet B was selected over Inlet A for the subcritical contraction design since the simulations indicated a decrease in flow separation with Inlet B. Inlet B should be easier to construct in field applications and should therefore be more cost effective than Inlet $\mathrm{C}$, although hydraulically both inlets performed equally well in simulations.

\section{Throat Length Design}

A primary consideration in the design of the subcritical contraction is its cost. Contraction length is proportional to construction

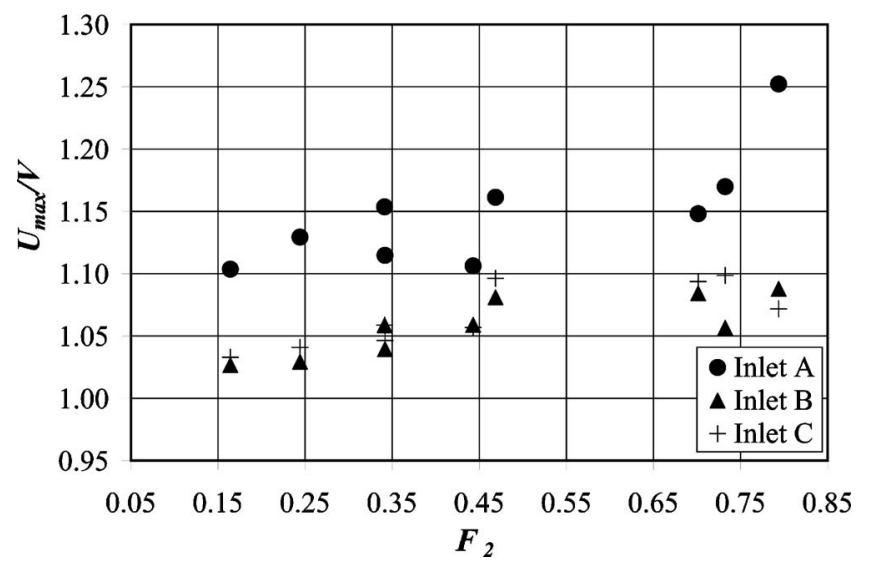

Fig. 3. Comparison of $U_{\max } / V$ at measurement location $\mathrm{M} 2$ at the downstream end of each inlet design tested 

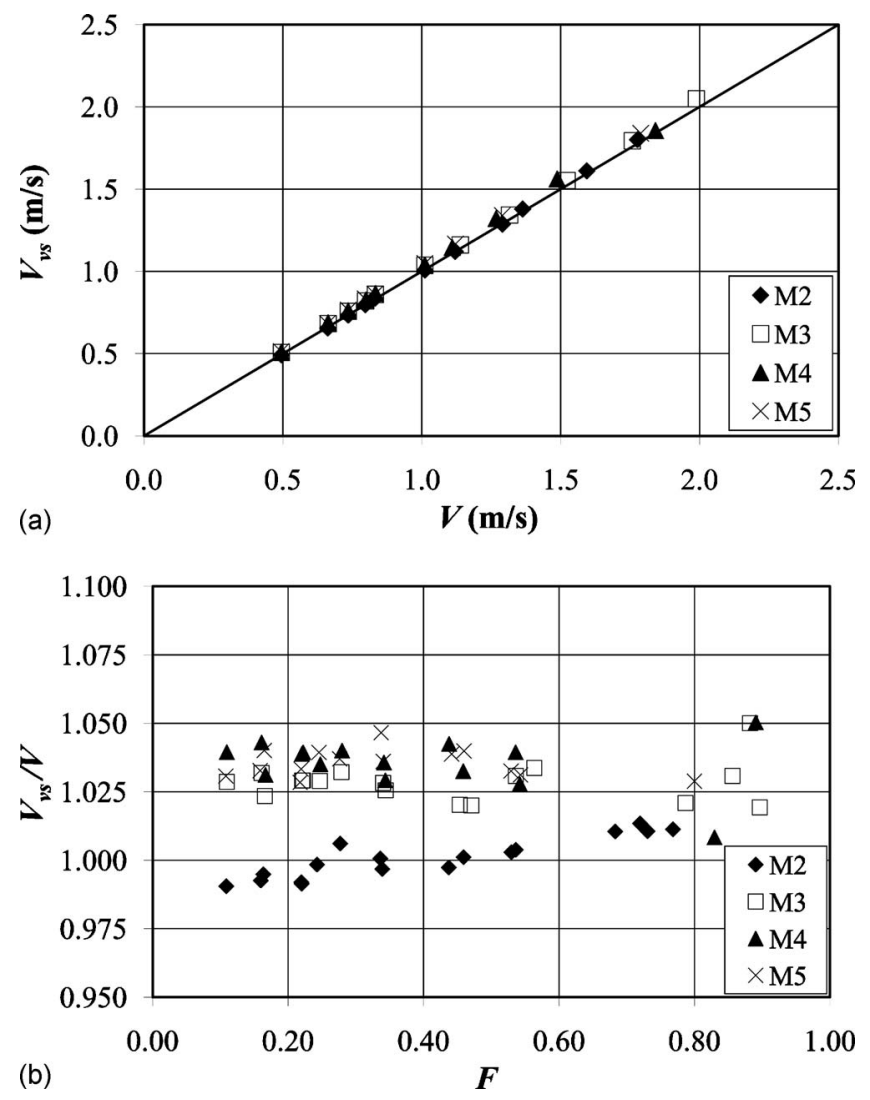

Fig. 4. Sampled velocities along the length of the contraction, (a) comparing $V_{v s}$ to $V$ at each measurement location; (b) $V_{v s} / V$ versus $\mathrm{F}$ calculated at each location

cost; therefore, a shorter RVF contraction results in lower construction cost. The first analysis examines the vertical velocity distribution at different central locations along the contraction throat. Inlet B was simulated with a throat length of $4 \mathrm{~m}$. The same flow rate and depth scenarios from the inlet design analysis were used for these simulations. Twelve sample velocity points were extracted at M2, M3, M4, and M5 (Fig. 2) spaced evenly along the vertical centerline.

Initial analysis examined the relationship of average vertical sample velocity $\left(V_{v s}\right)$ to $V$ at measurement locations M2-M5 shown in Fig. 4. The comparison in Fig. 4(a) shows a relationship between the average sample velocity and the cross-sectional averaged velocity at each measurement location suggesting that measurements could be made at any location within the contraction throat. Interestingly, from Fig. 4(b), the ratio of $V_{v s} / V$ at M2 was very close to 1 for each of the scenarios analyzed.

The next analysis examined whether throat length affected vertical centerline velocities. A comparison was made for simulated Inlet B contractions using throat lengths of 1 and $4 \mathrm{~m}$. Comparing the vertical centerline velocities at key locations within the two significantly different throat lengths should provide an indication of the impact of throat length. Flow rate and depth scenarios included discharges of $0.283,0.425,0.566$, and $0.708 \mathrm{~m}^{3} / \mathrm{s}$ at nominal depths of 1 and $0.65 \mathrm{~m}$ as well as $0.283 \mathrm{~m}^{3} / \mathrm{s}$ at a nominal depth of $0.35 \mathrm{~m}$.

Vertical velocities were sampled at 12 evenly spaced intervals from the channel bottom to the water surface from the M2 and M3 locations and compared for the two contraction lengths. The ratios of average vertical sample velocity $\left(V_{v s}\right)$ to average cross-

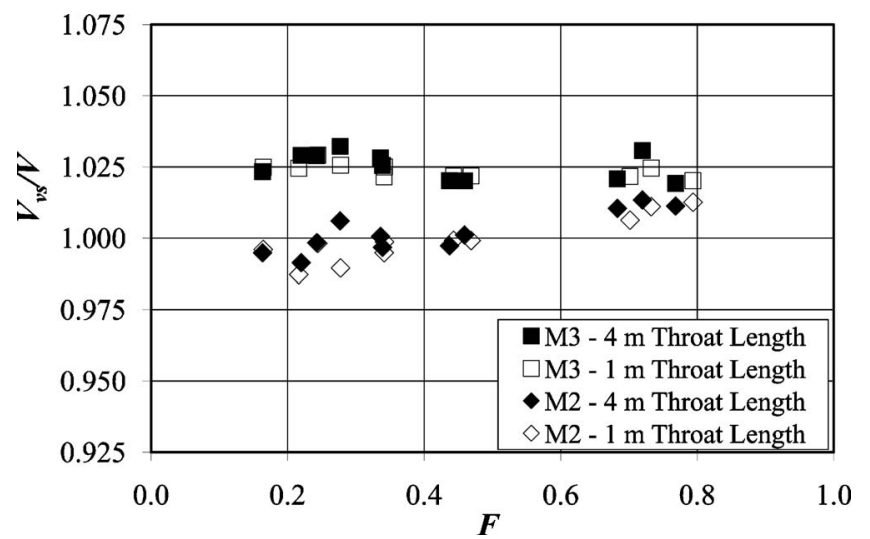

Fig. 5. Comparison of $V_{v s} / V$ by $\mathrm{F}$ for a throat length of 4 and $1 \mathrm{~m}$ at $\mathrm{M} 2$ and M3

sectional velocity $(V)$ were plotted against the $\mathrm{F}$ in Fig. 5 for throat lengths of 1 and $4 \mathrm{~m}$. The results indicate minimal difference in sample velocities between the two throat lengths at either M2 or M3, suggesting no advantage for a longer throat.

Since length of the contraction throat did not significantly impact the vertical velocity profile, the final consideration for throat length design is the configuration of the upward-looking ADVM within the contraction. Because the upward-looking ADVM takes velocity measurements along specific beam angles and those measurements must be taken within the contraction throat, the length of the throat is a function of the upward-looking ADVM beam angle $\left(\theta_{\text {beam }}\right)$ and the maximum possible flow depth $\left(h_{\text {max }}\right)$. The maximum distance between the beams using $\theta_{\text {beam }}$ of $45^{\circ}$ is approximately $2 h_{\max }$. Therefore, the subcritical RVF contraction throat length $\left(L_{T L}\right)$ is set equal to $2 h_{\max }$ for the $45^{\circ}$ beam angled ADVM and the ADVM should be placed at a distance equal to $h_{\text {max }}$ downstream of the start of the throat to ensure that the ADVM beam remains within the throat.

\section{Contraction Ratio Design}

The effect of the CR on repeatability and consistency of the centerline vertical velocity profile was tested by simulating two CRs. Using Inlet B with a throat length of $2 \mathrm{~m}$, the CRs of 0.5 and 0.75 were modeled to develop a range of the Froude numbers and to examine the impact of $\mathrm{F}$ on the relationship between an average theoretical ADVM sample velocity $\left(V_{\mathrm{T}-\mathrm{ADVM}}\right)$ and $V$. Since the design of Inlet $\mathrm{B}$ is a function of the width of the contraction opening, $b$, the inlet was modified based on the design criteria in Fig. 1 for the different CRs analyzed. For CRs of 0.5 and $0.75, b$ equals 0.6075 and $0.9112 \mathrm{~m}$, respectively, for the simulated contractions.

Velocity samples were extracted from the simulation results along the theoretical ADVM beams ( $\theta_{\text {beam }}$ equal to $45^{\circ}$ from vertical) from $0.15 \mathrm{~m}$ vertically above the channel bottom at depths every $0.034 \mathrm{~m}$ to just below the water surface. The buffer distance $\left(z_{b}\right)$ of $0.15 \mathrm{~m}$ above the channel bottom accounts for an ADVM and mount height of $0.08 \mathrm{~m}$ and a blanking distance above the ADVM of $0.07 \mathrm{~m}$ so that the readings are outside of any flow disturbance caused by the device. Buffer distances can vary depending on how the device is mounted. The extracted velocities were averaged to develop the theoretical ADVM velocity $\left(V_{\mathrm{T}-\mathrm{ADVM}}\right)$. The Froude number was calculated for each scenario using $Q$ into the simulated flume, $b$ for each scenario, and the 
Table 1. Contraction Ratios Simulated for Each Flow Rate and Depth Scenario

\begin{tabular}{lccc}
\hline & \multicolumn{3}{c}{$\begin{array}{c}\text { Nominal depth } \\
(\mathrm{m})\end{array}$} \\
\cline { 2 - 4 } $\begin{array}{l}\text { Discharge } \\
\left(\mathrm{m}^{3} / \mathrm{s}\right)\end{array}$ & 1.00 & 0.65 & 0.35 \\
\hline 0.283 & $0.5,0.75$ & $0.5,0.75$ & $0.5,0.75$ \\
0.425 & $0.5,0.75$ & $0.5,0.75$ & 0.75 \\
0.566 & $0.5,0.75$ & $0.5,0.75$ & $\mathrm{~F} \geq 1$ \\
0.708 & $0.5,0.75$ & 0.75 & $\mathrm{~F} \geq 1$ \\
\hline
\end{tabular}

flow depth $(h)$ at the theoretical ADVM location. The CFD testing scenarios used for the CR analysis are listed in Table 1.

The results of the $\mathrm{CR}$ analysis are shown in Fig. 6. Interestingly, there was no evident difference in $V_{\mathrm{T}-\mathrm{ADVM}} / V$ between the 0.5 and $0.75 \mathrm{CRs}$; however, there was increased variability in $V_{\mathrm{T}-\mathrm{ADVM}} / V$ for both $\mathrm{CRs}$ at higher $\mathrm{F}$. The increased variability in $V_{\mathrm{T}-\mathrm{ADVM}} / V$ at higher $\mathrm{F}$ is likely due to an uneven water surface due to standing waves within the flow measurement section caused by the higher velocities. This indicates that there could be increased error if the $\mathrm{CR}$ is set below some optimal level resulting in the high Froude numbers.

The CR must be designed based on-site-specific parameters such as discharge, channel width, and flow depth. The design criteria will provide minimum and maximum CRs that should be considered, allowing the designer to select the CR that is most appropriate. In order to develop a sufficient velocity profile disturbance to maintain a direct relationship between the ADVM sample velocity and the average cross sectional velocity, a maximum $\mathrm{CR}\left(\mathrm{CR}_{\max }\right)$ should be 0.75 . The minimum $\mathrm{CR}\left(\mathrm{CR}_{\min }\right)$ and minimum contraction opening $\left(b_{\min }\right)$ are a function of the maximum Froude number $\left(\mathrm{F}_{\max }\right)$. Because of increased variability in $V_{\mathrm{T}-\mathrm{ADVM}} / V$ at $\mathrm{F}$ (Fig. 6) and the potential for wave formation within the measurement section, $\mathrm{CR}_{\min }$ and $b_{\text {min }}$ can be calculated for $F_{\text {max }}=0.50$ as Eqs. (2) and (3)

$$
\mathrm{CR}_{\text {min }}=\frac{Q_{\text {max }}}{0.50 B h_{\text {min }}^{3 / 2} g^{1 / 2}}
$$

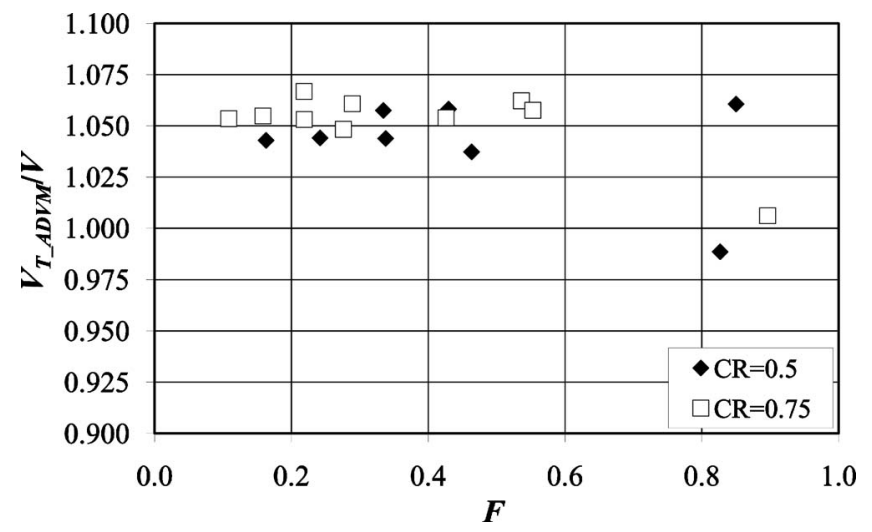

Fig. 6. Comparison of $V_{\mathrm{T}-\mathrm{ADVM}} / V$ versus $\mathrm{F}$ for subcritical contraction with Inlet $\mathrm{B}$, throat length of $2 \mathrm{~m}$, and a theoretical ADVM with a $45^{\circ}$ beam angle

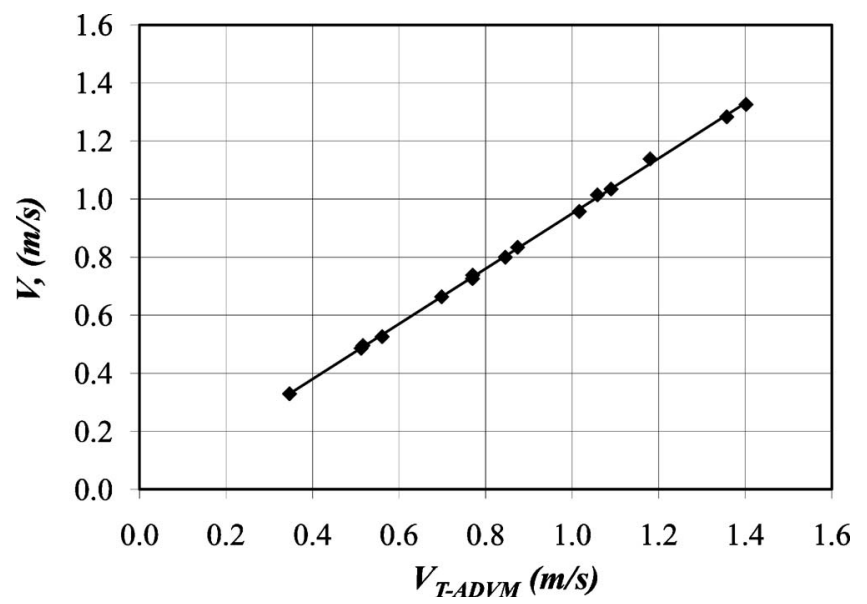

Fig. 7. Relating $V_{\mathrm{T}-\mathrm{ADVM}}$ to $V$ for an upward-looking ADVM with $z_{b}=0.15 \mathrm{~m}$

$$
b_{\text {min }}=\frac{Q_{\max }}{0.50 h_{\text {min }^{*}}^{3 / 2} g^{1 / 2}}
$$

where $Q_{\max }=$ maximum expected flow rate in the channel; $h_{\min }{ }^{*}$ $=$ minimum flow depth in the channel at the maximum flow rate; $B=$ upstream channel width measured from bank to bank at $50 \%$ of the maximum depth; and $g=$ gravitational acceleration.

Of course, actual $h_{m i n} *$ will be lower in the contraction caused by the conversion of potential energy to kinetic energy. This will cause the actual Froude number to be greater than 0.5 if $\mathrm{CR}_{\min }$ or $b_{\text {min }}$ is selected for the design. Ideally, the design CR and $b$ will be selected between $\mathrm{CR}_{\text {max }}$ and $\mathrm{CR}_{\text {min }}$ so that $\mathrm{F}_{\text {max }}$ is $0.15-0.36$ to obtain the highest degree of accuracy. In special cases where $\mathrm{CR}_{\text {min }}$ is selected for the subcritical contraction design, $\mathrm{CR}_{\text {min }}$ can be corrected through an iterative process using the conservation of energy principle solving for the water depth in the contraction $\left(h_{\text {min }}\right.$ ) using the initial $\mathrm{CR}_{\text {min }}$ estimate. CFD simulations indicate that flow streamlines within the contraction throat are nearly parallel near the design location of the ADVM, so therefore hydrostatic pressure distribution can be assumed near the middle of the contraction at a minimum depth of $0.35 \mathrm{~m}$.

\section{Computed Cross-Sectional Average Velocity}

In order to relate the upward-looking ADVM measured velocity $\left(V_{\mathrm{ADVM}}\right)$ to the cross-sectional average velocity $(V)$ within the 2-m contraction a relationship was developed using the simulated results from the scenarios listed in Table 1. Velocity data were extracted from simulation results along theoretical ADVM beams for $\theta_{\text {beam }}$ of $45^{\circ}$ and averaged to develop $V_{\text {T-ADVM }}$. Since upwardlooking ADVM mountings can differ, the distance above the channel bottom to the first measurement (buffer distance) varies by installation. Buffer distances $\left(z_{b}\right)$ of $0.082,0.116,0.15$, and $0.184 \mathrm{~m}$ were used for each simulated scenario to analyze its impact.

A linear relationship was found to exist between $V_{\mathrm{T}-\mathrm{ADVM}}$ and $V$ for each $z_{b}$ in the form of Eq. (4)

$$
V=C_{s c} V_{\mathrm{ADVM}}
$$

where $C_{s c}$ (the subcritical contraction coefficient) is dependent on the ADVM beam angle and $z_{b}$. Fig. 7 shows the regression analysis for the $z_{b}$ of $0.15 \mathrm{~m}$. The linear regression on the data from the Fig. 7 resulted in a $C_{s c}=0.9499$ with an $r^{2}=0.9995$. 
Table 2. Subcritical Contraction Coefficient and the Linear $r^{2}$ Value for Each Buffer Distance Analyzed $\theta_{\text {beam }}$ of $45^{\circ}$

$\begin{array}{lcc}z_{b} & & r_{s c} \\ (\mathrm{~m}) & 0.9488 & 0.9994 \\ 0.184 & 0.9499 & 0.9995 \\ 0.150 & 0.9514 & 0.9995 \\ 0.116 & 0.9539 & 0.9996 \\ 0.082 & \end{array}$

The $C_{s c}$ values developed using the regression analysis for all $z_{b}$ and beam angles are shown in Table 2 along with their respective $r^{2}$ values. A polynomial line fitting was used to develop the following relationships for $C_{s c}$ as a function of $z_{b} / z_{b \text { max }}$ as shown in Eq. (5):

$$
C_{s c}=0.0121\left(\frac{z_{b}}{z_{b \max }}\right)^{2}-0.026\left(\frac{z_{b}}{z_{b \text { max }}}\right)+0.9625
$$

where $z_{b}=$ distance from the channel bottom to the first measurement in meters and $z_{b \text { max }}=$ maximum buffer distance equal to 0.2 $m$ that should be used with these equations. The minimum buffer distance $\left(z_{b \text { min }}\right)$ should not be set below $0.08 \mathrm{~m}$. The $r^{2}$ value for the $C_{s c}$ equations using the simulated data was 0.9988 .

Changes in the side-wall boundary layers, through the transition, are likely the reason for the consistency in the relationship between $V$ and $V_{\mathrm{ADVM}}$. Further analysis is underway to determine how and what type of effects the contraction has on the boundary layer.

\section{Summary of the Subcritical Contraction Design}

The final design of the subcritical contraction is presented in Fig. 8. The design utilizes Inlet B from Smith (1967) where the radius of curvature $\left(R_{\text {entrance }}\right)$ for the $33.7^{\circ}$ rounded portion of the entrance is calculated as Eq. (6)

$$
R_{\text {entrance }}=1.65(B-b)
$$

where $b=$ contraction opening width calculated from Eq. (3) and $B=$ channel width for a rectangular channel or the channel width at $50 \%$ of the maximum flow depth $\left(h_{\max }\right)$. The throat length is also a function of the $h_{\max }$ calculated as Eq. (7)

$$
L_{T L}=2 h_{\max }
$$

where $h_{\max }=$ maximum flow depth expected from the channel bottom to the water surface at the flow measurement site before contraction installation.

The location of the upward-looking ADVM $\left(L_{\mathrm{ADVM}}\right)$ relative to the throat entrance is a function of the device beam angle and $h_{\text {max }}$ calculated as Eq. (8)

$$
L_{\mathrm{ADVM}}=h_{\text {max }} \tan \left(\theta_{\text {beam }}\right)
$$

where $\theta_{\text {beam }}=\mathrm{ADVM}$ beam angle. For $45^{\circ} \theta_{\text {beam }}, \tan \left(\theta_{\text {beam }}\right)$ is equal 1.0 .

The exit transition shown in Fig. 8 is a simple rounded exit with a radius calculated as

$$
R_{\text {exit }}=\frac{(B-b)}{2}
$$

The exit condition has not been shown to impact the upwardlooking ADVM accuracy; therefore, the designer may choose an angled exit wall instead of the rounded transition.

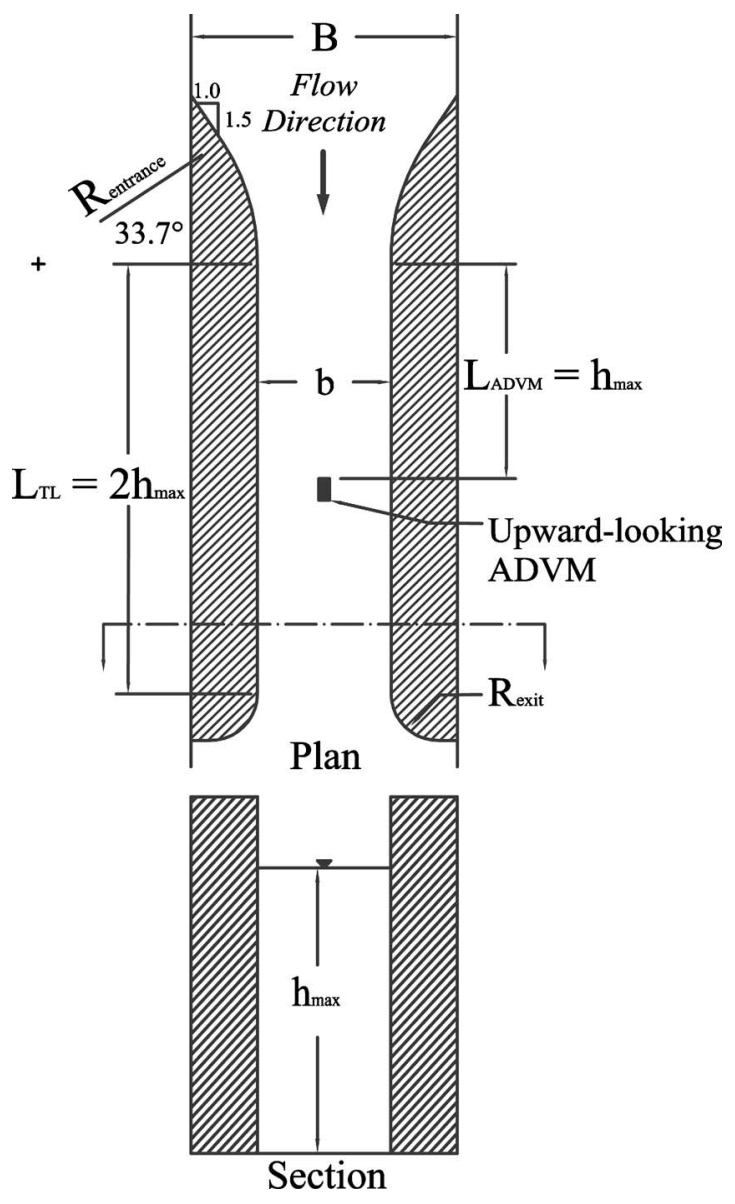

Fig. 8. Final design of the subcritical contraction showing a plan and section for improved upward-looking ADVM accuracy with flow moving from top to bottom in the plan view

Several other design parameters impact the effectiveness of the subcritical contraction. The minimum water depth of $0.3 \mathrm{~m}$ above the installed device is necessary to obtain accurate data from the ADVM. The subcritical contraction walls and floor should be relatively smooth (e.g., finished concrete or noncorrugated steel) and painted with special marine quality paint to prevent aquatic growth. The height of the contraction should be set with sufficient freeboard so that overtopping does not occur. It may be necessary to have the top of the contracted sidewalls at the same elevation as the top of the channel bank. The floor of the contraction should be at the same elevation as the existing channel bed.

\section{Contraction in Trapezoidal Channel}

To validate how the subcritical contraction would perform in a trapezoidal channel, a trapezoidal channel was modeled in the CFD software. The CFD trapezoidal channel had a bottom width of $0.6075 \mathrm{~m}$, a side slope of 0.6075:1 (horizontal:vertical), a depth of $1.2 \mathrm{~m}$, and a channel bottom slope of 0.002 . These dimensions resulted in the same final subcritical contraction design, with a CR of 0.5 used for the rectangular channel. Six tests were simulated using the trapezoidal channel and subcritical contraction with discharge rates of 0.283 and $0.425 \mathrm{~m}^{3} / \mathrm{s}$ at nominal depths of 0.65 and $1 \mathrm{~m}$ and discharge rates of 0.566 and 0.708 $\mathrm{m}^{3} / \mathrm{s}$ at a nominal depth of $1 \mathrm{~m}$.

Testing involved extracting velocities along the theoretical ADVM beam angles to develop $V_{\mathrm{T}-\mathrm{ADVM}}$ as previously described 
Table 3. Results of the Trapezoidal Channel Simulation with the Subcritical Contraction Using Eq. (5) for $C_{s c}$ Based on $z_{b}$ and Eq. (4) for $V_{c}$

\begin{tabular}{|c|c|c|c|c|c|c|c|}
\hline $\begin{array}{l}Q \\
\left(\mathrm{~m}^{3} / \mathrm{s}\right)\end{array}$ & $\begin{array}{c}h \\
(\mathrm{~m})\end{array}$ & $\mathrm{F}$ & $\begin{array}{c}z_{b} \\
(\mathrm{~m})\end{array}$ & $\begin{array}{l}V_{\mathrm{T}-\mathrm{ADVM}} \\
(\mathrm{m} / \mathrm{s})\end{array}$ & $\begin{array}{c}V_{c} \\
(\mathrm{~m} / \mathrm{s})\end{array}$ & $\begin{array}{c}V \\
(\mathrm{~m} / \mathrm{s})\end{array}$ & $\begin{array}{c}\text { Percent error } \\
(\%)\end{array}$ \\
\hline 0.283 & 0.908 & 0.172 & 0.150 & 0.538 & 0.511 & 0.513 & -0.46 \\
\hline 0.425 & 0.931 & 0.249 & 0.150 & 0.784 & 0.745 & 0.752 & -0.92 \\
\hline 0.566 & 0.915 & 0.340 & 0.150 & 1.070 & 1.016 & 1.018 & -0.21 \\
\hline 0.708 & 0.841 & 0.482 & 0.150 & 1.497 & 1.422 & 1.386 & 2.65 \\
\hline 0.283 & 0.559 & 0.356 & 0.150 & 0.896 & 0.851 & 0.833 & 2.06 \\
\hline 0.425 & 0.561 & 0.532 & 0.150 & 1.323 & 1.257 & 1.247 & 0.76 \\
\hline 0.283 & 0.908 & 0.172 & 0.116 & 0.537 & 0.511 & 0.513 & -0.42 \\
\hline 0.425 & 0.931 & 0.249 & 0.116 & 0.783 & 0.745 & 0.752 & -0.86 \\
\hline 0.566 & 0.915 & 0.340 & 0.116 & 1.069 & 1.017 & 1.018 & -0.15 \\
\hline 0.708 & 0.841 & 0.482 & 0.116 & 1.494 & 1.422 & 1.386 & 2.60 \\
\hline 0.283 & 0.559 & 0.356 & 0.116 & 0.893 & 0.850 & 0.833 & 1.93 \\
\hline 0.425 & 0.561 & 0.532 & 0.116 & 1.320 & 1.256 & 1.247 & 0.68 \\
\hline
\end{tabular}

for $z_{b}$ of 0.116 and $0.15 \mathrm{~m}$. Eq. (5) was used to calculate $C_{s c}$ based on $z_{b}$. Eq. (4) was used to estimate the cross-sectional average velocity $\left(V_{c}\right)$ based on $V_{\text {T-ADVM. }}$. The actual average crosssectional velocity $(V)$ at the theoretical ADVM site was computed using the known flow rate $Q$ and the wetted area computed from the contraction width and measured $h$ at the ADVM location.

The results of the subcritical RVF contraction simulations in the trapezoidal channel are shown in Table 3. The results show relative errors between $V_{c}$ and $V$ ranging from -0.92 to $2.65 \%$ for a $z_{b}$ of $0.15 \mathrm{~m}$ and -0.86 to $2.60 \%$ for $z_{b}$ of $0.116 \mathrm{~m}$. There is some bias toward overestimating the velocity using Eq. (4). This bias is likely due to higher velocities in the upper portion (measurement range) of the flow profile, which was probably caused by hydraulic properties of the transition from a trapezoidal channel into the rectangular contraction. Since a larger volume of flow is near the water surface in a trapezoidal channel, as the flow converges into the rectangular subcritical contraction, the velocities toward the surface are greater than a rectangular channel to rectangular contraction. Nevertheless the range of error is considered very good from an open-channel flow measurement standpoint.

\section{Laboratory Flume Validation of Contraction Design}

The validation testing of the subcritical contraction involved physical measurements taken in the Cal Poly ITRC flume after the prototype geometry was manufactured and installed. The prototype design, shown in Fig. 8 with a CR of 0.5 and $L_{T L}$ equal to $2 \mathrm{~m}$, was installed in the Cal Poly ITRC flume 30-m downstream from the start of the flow measurement section. A Sontek/YSI Argonaut SW (SonTek SW), an upward-looking ADVM with a $\theta_{\text {beam }}$ of $45^{\circ}$, was installed within the contraction at $L_{\mathrm{ADVM}}$ equal to $1.0 \mathrm{~m}$.

Contraction validation involved analyzing flow rates varying between 0.132 and $0.571 \mathrm{~m}^{3} / \mathrm{s}$ with depths between 0.63 and 1.05 $\mathrm{m}$. The Froude number ranged from 0.11 to 0.49 for the physical testing. Buffer distances of 0.144 and $0.178 \mathrm{~m}$ were evaluated.

Velocity data from the SonTek SW were extracted at 3.4-cm intervals starting at the buffer distance $\left(z_{b}\right)$ to the closest interval below the water surface. Velocities at each depth were averaged over 5-min intervals and recorded by the ADVM. The number of 5-min average samples for each testing scenario ranged from 20 to 50. $V_{\mathrm{ADVM}}$ was computed as the average of the velocity values from the buffer distance to just below the water surface for each 5-min sample. The estimated cross sectional average velocity $\left(V_{c}\right)$ was computed using Eq. (10)

$$
V_{c}=C_{s c} V_{\mathrm{ADVM}}
$$

The actual cross-sectional velocity $(V)$ was calculated based on the discharge from the Magmeter, SonTek SW water level, and contraction width at the ADVM location. Flow rates were sampled every $2 \mathrm{~s}$ and averaged over the same 5-min period as the SonTek SW. The relative error between $V_{c}$ and $V$ was computed using Eq. (11)

$$
\text { relative error }=\frac{\left(V_{c}-V\right)}{V} \times 100 \%
$$

\section{Results}

Results from tests with the subcritical contraction installed in the Cal Poly ITRC flume are shown for a $z_{b}$ of 0.144 and $0.178 \mathrm{~m}$ in Table 4. For each set of 5-min samples, the mean error as well as the upper and lower confidence limits of error within the sample population is shown based on a $99 \%$ confidence interval. The mean relative errors for each testing scenario ranged from -3.68 to $3.07 \%$ for $z_{b}=0.144 \mathrm{~m}$ and -3.41 and $3.31 \%$ for $z_{b}$ $=0.178 \mathrm{~m}$. The $99 \%$ confidence limits for $z_{b}$ of $0.144 \mathrm{~m}$ were within -3.86 and $3.25 \%$. This range was slightly higher for $z_{b}$ $=0.178 \mathrm{~m}$ with the minimum errors of -3.59 and $3.50 \%$ combining all testing scenarios.

For scenarios where $\mathrm{F}$ is less than 0.36 the mean relative error was less than $\pm 1 \%$. The increase in relative error for tests with larger $\mathrm{F}$ is due to standing wave formation within the contraction. Since $V$ is calculated from the discharge measured by the MagMeter, the contraction width $(b)$, and the flow depth measured by the ADVM, errors in the depth measurement due to wave formation show up in the error analysis. While a bias within each of the tests with $F$ greater than 0.3 is evident (e.g., the $0.440-\mathrm{m}^{3} / \mathrm{s}$ test with an $\mathrm{F}$ of 0.46 resulted in a mean relative error of $-3.68 \%$ with the upper and lower confidence intervals of -3.5 and $-3.86 \%$ ), there is no indication of bias toward overestimation or underestimation of velocity between the three tests. This indicates that the location of the standing waves moves relative to the ADVM depending on F.

Fig. 9 shows a comparison between the velocity distribution within the subcritical RVF contraction using the average velocity from the upstream and downstream ADVM beams computed 
Table 4. Results of Subcritical Validation Testing in the Cal Poly ITRC Flume for $z_{b}=0.144$ and $0.178 \mathrm{~m}$ Showing the Mean Relative Error of All Samples as well as the Range of Relative Errors for the 5-min Samples with a 99\% Confidence Level

\begin{tabular}{|c|c|c|c|c|c|c|c|c|c|c|}
\hline \multirow{2}{*}{$\begin{array}{l}\text { Average } \\
\text { discharge } \\
\left(\mathrm{m}^{3} / \mathrm{s}\right)\end{array}$} & \multirow[b]{2}{*}{$\begin{array}{c}z_{b} \\
(\mathrm{~m})\end{array}$} & \multirow{2}{*}{$\begin{array}{l}\text { Number } \\
\text { of 5-min } \\
\text { samples }\end{array}$} & \multicolumn{5}{|c|}{ Average } & \multirow{2}{*}{$\begin{array}{l}\text { Mean } \\
\text { relative } \\
\text { error of } V_{c} \\
\quad(\%)\end{array}$} & \multicolumn{2}{|c|}{$\begin{array}{l}99 \% \text { confidence } \\
\text { interval of error }\end{array}$} \\
\hline & & & $\begin{array}{l}\text { Depth } \\
\text { (m) }\end{array}$ & $\mathrm{F}$ & $\begin{array}{l}V_{\mathrm{ADVM}} \\
(\mathrm{m} / \mathrm{s})\end{array}$ & $\begin{array}{c}V_{c} \\
(\mathrm{~m} / \mathrm{s})\end{array}$ & $\begin{array}{c}V \\
(\mathrm{~m} / \mathrm{s})\end{array}$ & & $\begin{array}{c}\text { Upper } \\
(\%)\end{array}$ & $\begin{array}{c}\text { Lower } \\
(\%)\end{array}$ \\
\hline 0.132 & 0.144 & 50 & 0.727 & 0.11 & 0.317 & 0.301 & 0.299 & 0.78 & 1.25 & 0.31 \\
\hline 0.292 & 0.144 & 30 & 0.926 & 0.17 & 0.543 & 0.516 & 0.518 & -0.32 & 0.03 & -0.68 \\
\hline 0.132 & 0.144 & 50 & 0.456 & 0.22 & 0.501 & 0.476 & 0.475 & 0.28 & 0.87 & -0.30 \\
\hline 0.440 & 0.144 & 50 & 1.000 & 0.23 & 0.767 & 0.728 & 0.722 & 0.88 & 1.02 & 0.75 \\
\hline 0.293 & 0.144 & 30 & 0.739 & 0.24 & 0.682 & 0.648 & 0.651 & -0.36 & 0.01 & -0.74 \\
\hline 0.571 & 0.144 & 20 & 1.053 & 0.28 & 0.939 & 0.892 & 0.889 & 0.35 & 0.56 & 0.14 \\
\hline 0.294 & 0.144 & 50 & 0.647 & 0.30 & 0.784 & 0.744 & 0.746 & -0.21 & 0.07 & -0.48 \\
\hline 0.440 & 0.144 & 50 & 0.749 & 0.36 & 1.045 & 0.993 & 0.963 & 3.07 & 3.25 & 2.88 \\
\hline 0.440 & 0.144 & 50 & 0.631 & 0.46 & 1.159 & 1.101 & 1.143 & -3.68 & -3.50 & -3.86 \\
\hline 0.570 & 0.144 & 50 & 0.728 & 0.48 & 1.388 & 1.318 & 1.288 & 2.40 & 2.60 & 2.20 \\
\hline 0.132 & 0.178 & 50 & 0.727 & 0.11 & 0.318 & 0.302 & 0.299 & 1.03 & 1.52 & 0.54 \\
\hline 0.292 & 0.178 & 30 & 0.926 & 0.17 & 0.545 & 0.518 & 0.518 & -0.03 & 0.34 & -0.40 \\
\hline 0.132 & 0.178 & 50 & 0.456 & 0.22 & 0.506 & 0.480 & 0.475 & 1.11 & 1.71 & 0.51 \\
\hline 0.440 & 0.178 & 50 & 1.000 & 0.23 & 0.769 & 0.730 & 0.722 & 1.09 & 1.23 & 0.95 \\
\hline 0.293 & 0.178 & 30 & 0.739 & 0.24 & 0.686 & 0.651 & 0.651 & 0.01 & 0.41 & -0.39 \\
\hline 0.571 & 0.178 & 20 & 1.053 & 0.28 & 0.941 & 0.893 & 0.889 & 0.51 & 0.72 & 0.30 \\
\hline 0.294 & 0.178 & 50 & 0.647 & 0.30 & 0.784 & 0.744 & 0.746 & -0.32 & -0.05 & -0.60 \\
\hline 0.440 & 0.178 & 50 & 0.749 & 0.36 & 1.048 & 0.995 & 0.963 & 3.31 & 3.50 & 3.12 \\
\hline 0.440 & 0.178 & 50 & 0.631 & 0.46 & 1.163 & 1.104 & 1.143 & -3.40 & -3.21 & -3.59 \\
\hline 0.570 & 0.178 & 50 & 0.728 & 0.48 & 1.395 & 1.324 & 1.288 & 2.82 & 3.02 & 2.62 \\
\hline
\end{tabular}

from the CFD model and the measured test using similar flow rates and depths. The discharge and depths in the model run and the measured test were not equal so the velocities at each depth from the model were normalized to the $V_{\mathrm{ADVM}}$ from the measured test. The model scenario used had a discharge rate of $0.283 \mathrm{~m}^{3} / \mathrm{s}$ and $h$ of $0.94 \mathrm{~m}$. The measured test used for the comparison had an average discharge of $0.292 \mathrm{~m}^{3} / \mathrm{s}$ and $h$ of $0.926 \mathrm{~m}$. The normalized streamwise average depth velocities $(u)$ closely match at $z / h$ above 0.3 . At $z / h$ below 0.3 the measured velocity is less than the model. This could be due to turbulence caused by the upwardlooking ADVM impacting the lower velocity measurement or an underestimation of the bottom roughness in the model.

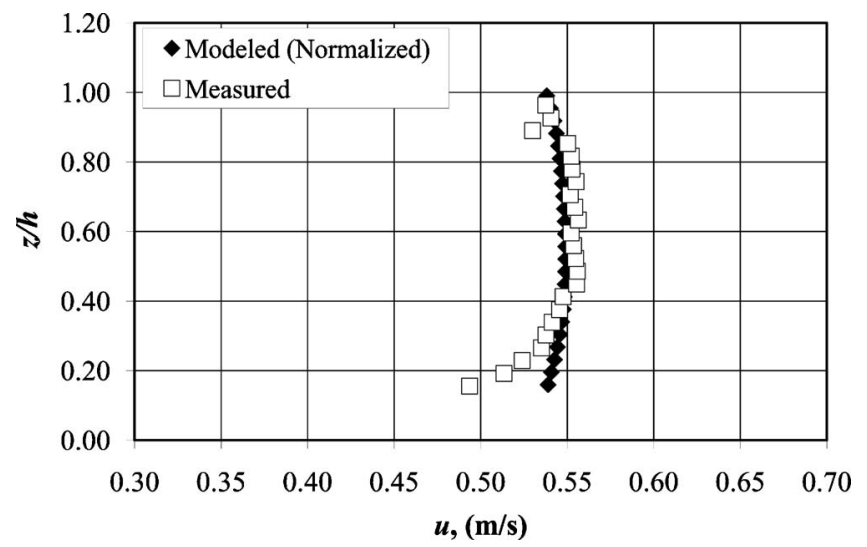

Fig. 9. Comparison of upward-looking ADVM measured velocity distribution to the normalized modeled velocity distribution extracted along theoretical ADVM beam angles

\section{Conclusion}

A subcritical contraction design was presented that can be used in conjunction with a vertical sampling upward-looking ADVM to provide accurate discharge measurement without in situ calibration. The subcritical RVF contraction design was validated using physical laboratory measurements taken at the Cal Poly ITRC flume, which is rectangular in cross section. Results indicate that the RVF contraction used in conjunction with the SonTek SW will provide accurate velocity measurement within $\pm 4 \%$ without calibration, with a $99 \%$ confidence level. This is an improvement to the $\pm 6 \%$ error using the conventional QIP method in a UF section with a recommended 10 calibration points (Styles et al. 2006). The results indicate that the error can be reduced to $\pm 2 \%$ (99\% confidence level) if $\mathrm{F}$ is below 0.36 . CFD testing suggests that a similar level of accuracy will be achieved when the contraction is placed in a trapezoidal channel but additional testing is warranted to quantify additional error.

\section{Acknowledgments}

Support for this research was provided by the U.S. Environmental Protection Agency under Grant No. EM-83335801. The writers gratefully acknowledge Mohamed Grissa for his participation in the laboratory experiments.

\section{Notation}

The following symbols are used in this paper:

$A=$ hydraulic or wetted cross-sectional area;

ADVM = acoustic Doppler velocity meter; 


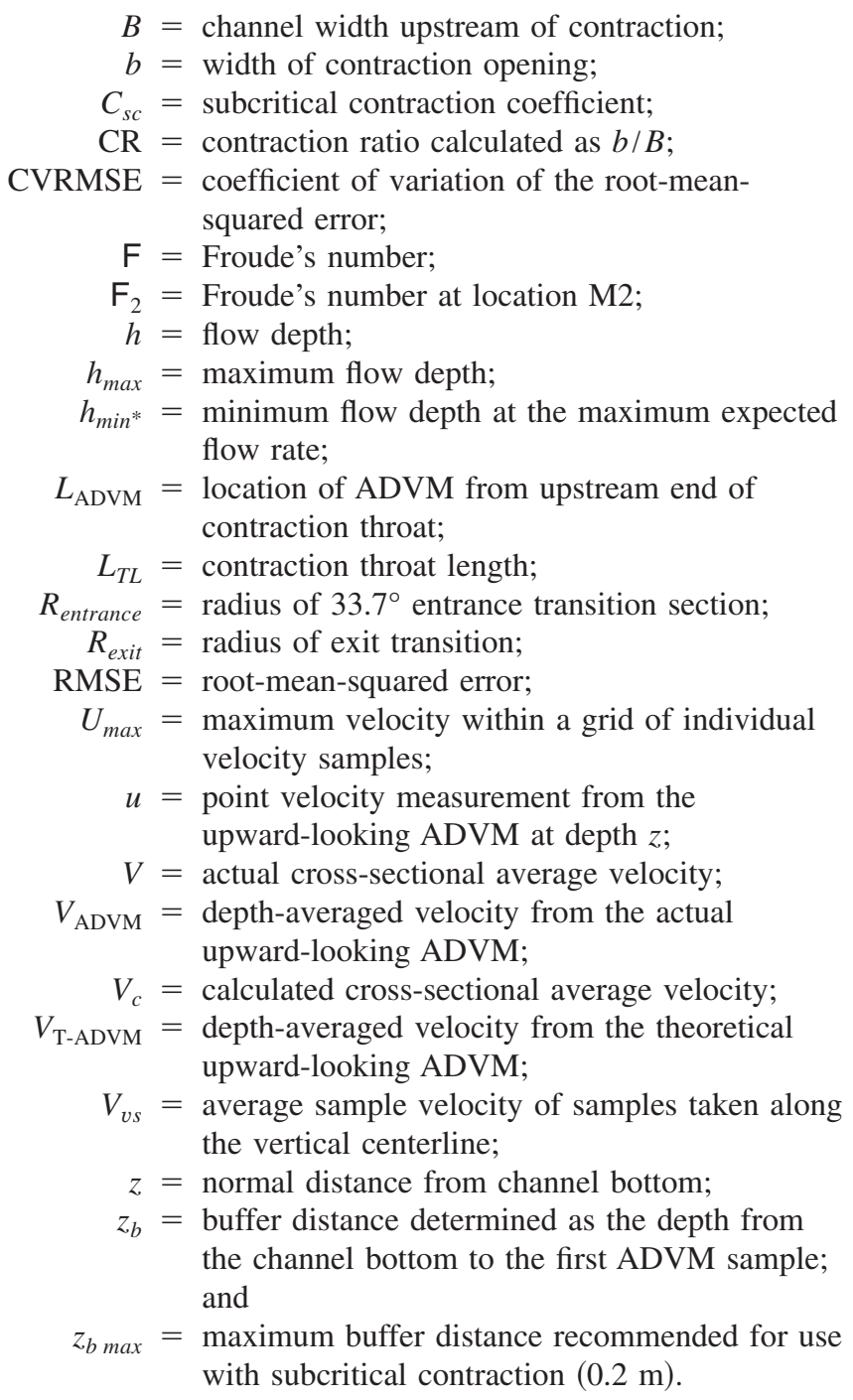

\section{References}

Chow, V. T. (1959). Open-channel hydraulics, McGraw-Hill, New York. Clemmens, A. J., and Bos, M. G. (1992). "Critical depth relations for flow measurement design.” J. Irrig. Drain. Eng., 118(4), 640-644.
Clemmens, A. J., Replogle, J. A., and Bos, M. G. (1984). "Rectangular measuring flumes for lined and earthen channels." J. Irrig. Drain. Eng., 110(2), 121-137.

Hardcastle, P. J. (1995). "A high resolution coherent acoustic Doppler profiler for the measurement of near bed turbulent flow." Conf. Proc., OCEANS '95 MTS/IEEE: Challenges of Our Changing Global Environment, Vol. 2, San Diego, 1361-1366.

Hardcastle, P. J., and Thorne, P. D. (1997). "Simultaneous measurements of high resolution vertical and horizontal flow profiles and suspended sediments using acoustic backscattering." Proc., 7th Int. Conf. on Technology Transfer from Research to Industry, Southampton, U.K., 100-106.

Huhta, C., and Ward, C. (2003). "Flow measurements using an upwardlooking Argonaut-SW Doppler current meter." Proc., IEEE/OES 7th Working Conf. on Current Measurement Technology, San Diego, 3539.

Irrigation Training and Research Center (ITRC). (2005). Hydroacoustic meters, Irrigation Training and Research Center, California Polytechnic State Univ., San Luis Obispo, Calif.

Montes, S. (1998). Hydraulics of open channel flow, ASCE, Baltimore.

Morlock, S. E., Nguyen, H. T., and Ross, J. H. (2002). "Feasibility of acoustic Doppler velocity meters for the production of discharge records from U.S. Geological Survey stream flow-gauging stations." 01-4157, U.S. Geological Survey, Indianapolis.

Patino, E., and Ockerman, D. (1997). "Computation of mean velocity in open channels using acoustic velocity meters." Open-File Rep. No. 97-220, U.S. Geological Survey, Tallahassee, Fla., 12.

Replogle, J. A. (2002). "Some observations on irrigation flow measurements at the end of the millennium." Appl. Eng. Agric., 18(1), 47-55.

Simpson, M. R. (2001). "Discharge measurements using a broad-band acoustic Doppler current profiler.” 01-01, U.S. Geological Survey, Sacramento, Calif.

Smith, C. D. (1967). "Simplified design for flume inlets." J. Hydr. Div., 93(HY6), 25-34.

Styles, S. W., Busch, B., Howes, D., and Cardenas, M. (2006). "Nonstandard structure flow measurement evaluation using the flow rate indexing procedure-QIP.” $R$ 06-003, Irrigation Training and Research Center, California Polytechnic State Univ., San Luis Obispo, Calif.

Vermeyen, T. B. (2000). "A laboratory evaluation of Unidata's Starflow Doppler flowmeter and MGD technologies' acoustic Doppler flowmeter." Proc., Joint Conf. on Water Resource Engineering and Water Resources Planning and Management, Minneapolis, ASCE, 318.

Vermeyen, T. B. (2005). "Laboratory evaluation of a shallow-water acoustic Doppler profiling flowmeter." Proc., World Water and Environmental Resources Congress, Anchorage, Alaska, 422. 